\title{
A Cross-sectional Survey of the North American Skull Base Society on Vestibular Schwannoma, Part 2: Perioperative Practice Patterns of Vestibular Schwannoma in North America
}

\author{
Jamie J. Van Gompel, MD ${ }^{1,2}$ Matthew L. Carlson, MD ${ }^{1,2}$
${ }^{1}$ Department of Otolaryngology-Head and Neck Surgery, Mayo Clinic School of Medicine, Rochester, Minnesota, United States
2 Department of Neurologic Surgery, Mayo Clinic School of Medicine, Rochester, Minnesota, United States
${ }^{3}$ Department of Otolaryngology-Head and Neck Surgery, Rush University Medical Center, Chicago, Illinois, United States
${ }^{4}$ Department of Otolaryngology-Head and Neck Surgery, Boston University School of Medicine, Boston Medical Center, Boston, Massachusetts, United States
${ }^{5}$ Department of Otolaryngology-Head \& Neck Surgery, Mayo Clinic College of Medicine, Phoenix, Arizona, United States
${ }^{6}$ Department of Neurological Surgery, University of Miami, Miller School of Medicine, Miami, Florida, United States

R. Mark Wiet, $\mathrm{MD}^{3} \quad$ Nicole M. Tombers, $\mathrm{RN}^{1}$

Anand K. Devaiah M, MD ${ }^{4}$ Devyani Lal, MD ${ }^{5}$ Jacques J. Morcos, MD $^{6}$ Michael J. Link, MD ${ }^{1,2}$

Address for correspondence Jamie J. Van Gompel, MD, FAANS, Division of Neurosurgery and Otorhinolaryngology, Department of Neurologic Surgery, Mayo Clinic, 200 First St. SW, Rochester, MN 55905, United States (e-mail: Vangompel.jamie@mayo.edu).

J Neurol Surg B 2018;79:297-301.

\begin{abstract}
Keywords

- vestibular schwannoma

- skull base

- cranial

- microsurgical

Introduction Perioperative care of vestibular schwannoma (VS) patients is extremely variable across surgeons and institutions making practice patterns difficult to standardize. No data currently exist detailing this practice variability.

Methods The North American Skull Base Society membership was electronically surveyed regarding perioperative care of surgically operated VS patients.

Results There were 87 respondents to the survey. Surgical positioning, surgical approach utilized, and perioperative medical adjuncts are quite variable. However, of those performing retrosigmoid approaches, $49 \%$ perform this in the supine position, while $33 \%$ use a park-bench position with only $2 \%$ using the sitting position. In those performing translabyrinthine approaches, $86 \%$ perform this in supine position. Although the use of neuromonitoring appears to be standard of care (98\%), other than the seventh nerve, there is substantial variability between respondents regarding monitoring of additional cranial nerves. Postoperative antibiotics are used by $65 \%$, postoperative steroids $81 \%$, and postoperative chemical deep vein thrombosis prophylaxis in $68 \%$ of survey respondents.

Conclusion Although the perioperative adjuncts to VS surgery are variable, there does appear to be a trend in common practice. Therefore, making surgeons aware of these trends may lead to standardized practice or alternatively trials of these variances to instruct which truly improve patient outcomes.
\end{abstract}

received

April 23, 2017

accepted after revision

September 19, 2017

published online

November 3, 2017 (c) 2018 Georg Thieme Verlag KG

Stuttgart · New York
DOI https://doi.org/

10.1055/s-0037-1607976. ISSN 2193-6331. 


\section{Introduction}

The perioperative details of current vestibular schwannoma (VS) surgery are not well described. First, there is no crosssectional study that has looked at the variance in patterns of surgical positioning, approach preference (translabyrinthine $[\mathrm{TL}]$ vs. retrosigmoid $[\mathrm{RS}]$ ), use of rigid cranial fixation, intraoperative procedural details, nor perioperative medical adjuncts. This study is part 2 of a cross-sectional electronic survey of the North American Skull Base Society (NASBS) to help define perioperative practice variance among surgeons who routinely perform VS microsurgical resection.

\section{Materials and Methods}

A 64-item Web-based survey assessing VS practice trends was devised by members of the NASBS Research Task Force and distributed to the NASBS membership via SurveyMonkey (-Appendix A, online only). Voluntary participation was solicited via e-mail with an attached electronic survey link available from November 29 through December 14, 2016. Following initial contact, survey reminders were sent 1 week and 24 hours before survey closure.

Responses were requested from those who are engaged in VS treatment. As a secondary screening measure, the first question of the survey inquired, "Are you actively involved in VS treatment at your center?" and the survey episode was subsequently closed for respondents who selected "no." All survey questions were multiple choice, and in most cases, survey items required selection of the single best answer. Respondent data were collected and compiled anonymously. Data from this large survey were apportioned into two separate reports according to topic: (1) overall management trends in VS across North America and (2) practice patterns of perioperative VS care in North America. This article presents data for the second of these two publications. Descriptive statistical analysis was performed using Microsoft Excel (Redmond, Washington, United States). Associations between features of interest were analyzed using Spearman's rank correlation, and comparisons between variables were assessed using KruskalWallis's, Wilcoxon's rank-sum, and Fisher's exact tests as appropriate. Statistical analyses were performed using version 9.4 of the SAS software package (SAS Institute, Cary, North Carolina, United States). All tests were two sided and $p$-values $<0.05$ were considered statistically significant. The Mayo Clinic Institutional Review Board deemed this study exempt from review. (In final text, plan will be to cite article of one of this series for methods.)

\section{Results}

\section{Survey}

There were 87 respondents to the survey; surveys were sent to 719 e-mails which are the whole membership of NASBS as of October 2016. This membership includes neurosurgeons, otologist, radiation oncologist, ophthalmologist, and plastic surgeons; therefore, the lower response rate likely has to do with responses only from those managing VS.
Table 1 Perioperative details of surgical approach

\begin{tabular}{|l|l|l|}
\hline Variable & Retrosigmoid & Translabyrinthine \\
\hline \multicolumn{2}{|l|}{ Position } & $5 \%$ \\
\hline $\begin{array}{l}\text { Lateral decubitus } \\
\text { (park bench) }\end{array}$ & $33 \%$ & $86 \%$ \\
\hline $\begin{array}{l}\text { Supine with head } \\
\text { turn (with or } \\
\text { without shoulder } \\
\text { bump) }\end{array}$ & $49 \%$ & $5 \%$ \\
\hline $\begin{array}{l}\text { Either, depending } \\
\text { on case }\end{array}$ & $14 \%$ & $0 \%$ \\
\hline $\begin{array}{l}\text { Sitting or } \\
\text { semisitting }\end{array}$ & $2 \%$ & $0 \%$ \\
\hline Prone & $2 \%$ & $4 \%$ \\
\hline $\begin{array}{l}\text { Not applicable, } \\
\text { Ido not use } \\
\text { this approach }\end{array}$ & $0 \%$ & $5 \%$ \\
\hline Cerebellar retraction & $21 \%$ & $5 \%$ \\
\hline $\begin{array}{l}\text { Always } \\
\text { At least } 50 \% \text { of } \\
\text { the time }\end{array}$ & $21 \%$ & $28 \%$ \\
\hline $\begin{array}{l}\text { Rarely } \\
\text { Never }\end{array}$ & $28 \%$ & $53 \%$ \\
\hline $\begin{array}{l}\text { I do not use } \\
\text { this approach }\end{array}$ & $28 \%$ & $5 \%$ \\
\hline Pinion use & & \\
\hline $\begin{array}{l}\text { Do you use } \\
\text { pinions? }\end{array}$ & & \\
\hline
\end{tabular}

\section{Perioperative Details of Surgical Approach}

-Table 1 provides a summary of details relative to differences between surgeon preferences for RS compared with TL approaches, middle fossa approaches were considered separately. In RS craniotomies (question 36), 49\% of surgeons utilized a supine position, $33 \%$ a lateral decubitus position, $14 \%$ vary between these two cases depending on the case, and only $2 \%$ reported utilizing a sitting position. Comparatively, in TL (question 37) cases, $86 \%$ of surgeons utilized a supine position, $5 \%$ a lateral decubitus position, and $5 \%$ varied between these two positions depending on the case. Surgeons reported the use of a cerebellar retractor routinely in RS cases $42 \%$ of the time (question 34), and in TL cases in only $14 \%$ of cases, while $53 \%$ never use cerebellar retraction in TL cases (question 35). Rigid fixation of the head, usually via three-point pinion fixation (question 33) was reported in $86 \%$ of RS operations, $26 \%$ of $\mathrm{TL}, 42 \%$ of middle fossa approaches, and $11 \%$ report no use of rigid head fixation.

An endoscope was not used (question 40) exclusively to remove a VS by any respondent. However, 37\% of surgeons reported using it in select situations such as when attempting hearing preservation, and 63\% never used an endoscope as an adjunct to the procedure. Tumor debulking was performed utilizing ultrasonic aspiration $83 \%$ of the time, tumor forceps and suction $9 \%$, scissors $5 \%$, and other microdebridment 
system $4 \%$. No surgeons reported utilizing a laser during VS resection (question 38). Antibiotic irrigation was used either most of the time (42\%) or sometimes (11\%); however, many surgeons (47\%) reported never using it (question 39).

\section{Closure}

RS craniotomies were closed most commonly with replacement of the native bone flap (58\%). Titanium mesh was used to replace a craniectomy defect $26 \%$ of the time, no reconstruction of the craniectomy was performed $4 \%$ of the time, and "other" was used in $12 \%$. Of interest, no respondents reported using bone cement (question 43). Following TL approaches, titanium mesh (25\%) was used most often to aid closure, followed by native bone (9\%), absorbable mesh (9\%), and bone cement was used $5 \%$ of the time (question 44 ). As expected, a fat graft was utilized in 95\% of TL cases. Primary approximation of dural leaflets was reported in $37 \%$ of cases, and $21 \%$ used artificial dural substitutes following TL surgery. Surgeons reported the following methods to prevent cerebrospinal fluid (CSF) leak associated with TL resection of VS: $65 \%$ pack the Eustachian tube with muscle or fascia at closure, $42 \%$ block the mastoid antrum with fascia, and $4 \%$ report oversewing the ear canal and packing the Eustachian tube at the time of primary surgery (question 44). Fifty-four percent surveyed report using a head wrap in the early postoperative phase after TL.

\section{Neuromonitoring}

Seventy-nine percent of respondents reported utilizing a separate neurophysiology team during surgery, while $21 \%$ utilized commercial products such as the NIMs (Medtronic Inc., Minneapolis, Minnesota, United States) monitor for cases which does not require a separate monitoring technician (question 24). When presented with a theoretical situation of a medium-sized VS $<2.5 \mathrm{~cm}$ (question 25) surgeons reported monitoring the trigeminal motor nerve (V3-Motor) in 32\% of cases, facial nerve $98 \%$ of the time, vagus nerve $(X) 12 \%$, spinal accessory nerve (XI) $12 \%$, and hypoglossal nerve (XII) in $4 \%$ of cases (see - Table 2 ). In surgeons performing hearing preserva-

Table 2 Neuromonitoring

\begin{tabular}{|l|l|l|}
\hline Variable & $\%$ & $N$ \\
\hline \multicolumn{2}{|l|}{ Nerve monitored for typical mid-sized VS } \\
\hline Facial nerve (VII) & $98 \%$ & 56 \\
\hline Trigeminal nerve (motor V) & $32 \%$ & 18 \\
\hline Vagus (X) & $12 \%$ & 7 \\
\hline Spinal accessory (XI) & $12 \%$ & 7 \\
\hline Hypoglossal (XII) & $4 \%$ & 2 \\
\hline Hearing monitoring in hearing preservation \\
\hline Far field 8 (BAER) & $72 \%$ & 41 \\
\hline Direct 8 (CNAP) & $16 \%$ & 9 \\
\hline No eighth nerve monitoring & $12 \%$ & 7 \\
\hline
\end{tabular}

Abbreviations: CNAP, cochlear nerve action potential, BAER, brain stem auditory evoked response; VS, vestibular schwannoma. tion surgery, $72 \%$ reported using far field eighth nerve monitoring (brain stem auditory evoked response), 16\% reported using direct eighth nerve monitoring (cochlear nerve action potentials), and $12 \%$ did not monitor the eighth nerve (see - Table 2 ).

\section{Immediate Postoperative Management}

Antibiotics were used $65 \%$ of the time postoperatively, while $35 \%$ reported never using postoperative antibiotics (question 61). In those who used postoperative antibiotics, $42 \%$ used them for only 24 hours, $23 \%$ for 1 to 3 days after surgery, $4 \%$ for 4 to 7 days after surgery, and $2 \%$ for more than a week (question 62). Steroids were prescribed (question 58) following removal of a VS most commonly for 4 to 7 days (32\%), for 8 to 14 days in $30 \%$ of patients, for 1 to 3 days in $19 \%$ of cases, and steroids were reportedly never used by $19 \%$ of surgeons who replied to the survey. Chemical deep vein thrombosis (DVT) prophylaxis was utilized in most patients ( $56 \%$ of the time). Also, in patients the surgeon deemed as "high risk" for DVT, an additional $12 \%$ of surgeons administered DVT prophylaxis. DVT prophylaxis was reported as rarely or never in $32 \%$ of respondents (question 59). DVT prophylaxis was initiated in those using it within 24 hours of surgery, $40 \%$ of the time; within 1 to 3 days, $28 \%$; within 4 to 7 days, 4\%; a week after surgery, $2 \%$; and never, $26 \%$ (question 60). CSF leaks (question 53) were managed mostly with a lumbar drain (77\%); however, conservative management (bed rest, lifting restrictions) was reported $9 \%$ of the time. Diamox was reported as being employed $2 \%$ of the time, and ear canal overclosure with packing of the Eustachian tube was reported by $12 \%$ of respondents to stop postoperative CSF leak as an initial treatment.

\section{Facial Nerve Management}

When a patient suffers profound facial weakness postoperatively, surgeons reported employing "aggressive" eye cares in $35 \%$ of cases as primary treatment for the symptoms of facial weakness, referred for upfront gold/platinum weight placement $49 \%$ of the time, and referred for upfront tarsorrhaphy $16 \%$ of the time (question 54). The perception of the cause of delayed facial weakness (question 55) was neural edema tracking up to the narrow labyrinthine segment of the facial nerve in $61 \%$, reactivation of latent virus $19 \%$, vasospasm in $18 \%$, and free pulsation of CSF on the cisternal segment of the facial nerve after tumor removal $2 \%$ of the time. Surgeons reported using retroviral therapy to treat delayed facial weakness (question 56) postoperatively most of the time in $14 \%$, sometimes ( $~ 50 \%)$ in $11 \%$, and rarely or never by $75 \%$ of the respondents.

\section{Prolonged Follow-up}

Thirty-seven percent of survey respondents reported getting their first postoperative magnetic resonance imaging (MRI) (question 63) $<48$ hours after tumor resection, within a month by $7 \%$ of the surgeon, between 1 and 6 months (37\%), between 7 and 12 months (9\%), and after 1 year, $11 \%$ of surgeons obtained a first postoperative MRI scan. After surgery, respondents reported allowing patients to return to work within 2 weeks, $4 \%$ of the time; at 1 month, $28 \%$; at 6 weeks, $47 \%$ of cases; at 3 months, $21 \%$; and no respondent routinely recommended return to work beyond 3 months (question 64). 


\section{Discussion}

Although we may feel within our own practices, there is substantial standardization of VS perioperative care, it is notable across the membership of the NASBS, there is considerable variability in practice. This phenomenon has been previously demonstrated with large registries such as the Surveillance, Epidemiology, and End Results database; however, data within these registries do not have granularity to look beyond surgical approach to assess these different practices. ${ }^{1}$ TL approaches appear to be the most standardized approaches. Most individuals utilize the supine position approximately $86 \%$ of the time. This may be secondary to the need for an abdominal fat graft and a decreased need to have the head turned very far to the contralateral side. Still, $5 \%$ of surgeons utilize the lateral decubitus position and another $5 \%$ vary depending on the case. Further, with TL procedures, there is consistently less utilization of static cerebellar retraction and only $26 \%$ of patients are placed in rigid fixation during TL procedures. There is more variability during RS craniotomies. Forty-nine percent report utilizing a supine position, while $33 \%$ use a lateral decubitus position. Interestingly, only $2 \%$ of NASBS surgeons responding to the survey reported using the sitting position for surgical resection of a VS.

Wound difficulties, and in particular CSF leak, are a significant concern for surgeons operating VS. Interestingly, there is significant variability in closure techniques following VS surgery. Following the TL approach, most surgeons use fat (95\% of the time) to fill the mastoidectomy defect, while $34 \%$ augment the fat by using some type of mesh over the fat graft. There is also considerable inconsistency in the pattern of which individuals attempt to block CSF leakage with $65 \%$ of respondents packing the Eustachian tube with muscle or fat and $42 \%$ simply blocking the antrum; however, only $4 \%$ oversew the ear canal at the time of primary tumor resection. Interestingly enough, not even a head wrap is standardized where approximately $50 \%$ of people doing TL approach utilized head wraps postoperatively. In terms of RS craniotomies, $58 \%$ of the time native bone flap is replaced, while $26 \%$ of the time, a titanium cranial mesh reconstruction is utilized. Therefore, despite good evidence that cranioplasty decreases postoperative headaches in VS, this is not standard of practice still. ${ }^{2-4}$ It is unknown as to whether or not these reconstruction techniques lead to differences in outcomes, however.

Neuromonitoring appears to be standard in that nearly $98 \%$ of individuals monitor the seventh nerve during resection. Most surgeons report utilizing a separate monitoring team (79\%), while $21 \%$ utilized commercial products such as a NIM monitor which are self-contained. ${ }^{5}$ In the case of a theoretical mediumsized tumor, the next most commonly assessed nerve was the motor portion of V3, however, only utilized in 32\% of cases. Additional monitoring such as the vagus nerve, spinal accessory nerve, or hypoglossal nerve was very infrequently utilized. Therefore, it appears to be standard of care to monitor the seventh nerve, although additional nerves are up to surgeon preference. In a situation of which one is performing hearing preservation surgery, $72 \%$ utilized auditory brain stem response (ABR) or far field eighth nerve monitoring, while only $16 \%$ report utilizing direct eighth nerve monitoring and cochlear nerve action potential. However, still 12\% did not utilize monitoring. The variance in these techniques may also be institutional in that some forms of monitoring may be dependent on the neurophysiologist teams, performing the monitoring alternatively may not be available at some institutions. ${ }^{6}$ Whether these are standard is a matter of debate as ABRs are often difficult to interpret intraoperatively and dropout well after hearing has changed, and further direct eighth nerve monitoring is not always possible depending on the position of the tumor. ${ }^{7,8}$

Wound infection and meningitis can be devastating complications associated with VS removal, regardless of size or technique. ${ }^{9}$ Interestingly, although, most would recommend antibiotics for at least a 24-hour period postoperatively, postoperative antibiotic use was only reported by $65 \%$ of surgeons, while $35 \%$ reported never utilizing postoperative antibiotics (-Table 3). There is variance, also, as to how long antibiotics were given postoperatively with $42 \%$ giving them for the traditional perioperative three doses in a 24 -hour period, while $4 \%$ reported 4 to 7 days or longer in some circumstances. This variance does not fall in line with most recent recommendations for standard practices care: Typically, the recommended peri-intraoperative dose is within 60 minutes of skin incision in addition to two postoperative doses of antibiotic covering skin flora. ${ }^{10}$ There currently exists no literature around perioperative antibiotics in VS cases; however, there are data to suggest bacitracin irrigation reduces rates of postoperative infection. ${ }^{11}$ Perioperative steroid use is very controversial, some use it to aid in controlling headaches and reduce inflammation of the seventh nerve; however, some choose to not utilize steroids for fear of secondary complications such as infection, poor wound healing, ulcers, and other medical consequences. Steroid use is very common postoperatively, although certainly not standard in this cohort. Only $19 \%$ of respondents did not use postoperative steroids; they were utilized most commonly for 4 to 7 days postoperatively. Perhaps, the most interesting of variable practice parameter assessed was DVT prophylaxis which was utilized $56 \%$ of the time, an additional $12 \%$ of surgeons reported using DVT prophylaxis in only high-risk patients, and 32\% reported never using chemical DVT prevention. Most surgeons reported using DVT prophylaxis within 24 hours of surgery. Very little data are available, currently, to suggest if there is an increased risk of perioperative hematoma formation with the use of postoperative chemical DVT prophylaxis. However, recent meta-analyses demonstrate probably a significant reduction in DVTs with a detectable increase in postoperative hematoma. ${ }^{12}$ Further, in this study, we did not assess the specific type of DVT prophylaxis utilized.

Table 3 Other perioperative adjuncts

\begin{tabular}{|l|l|}
\hline Question & Yes (\%) \\
\hline Do you use postoperative antibiotics? & 65 \\
\hline Do you use perioperative steroids? & 81 \\
\hline $\begin{array}{l}\text { Do you use perioperative chemical } \\
\text { DVT prophylaxis (including high risk)? }\end{array}$ & 68 \\
\hline
\end{tabular}

Abbreviation: DVT, deep vein thrombosis. 
Perhaps, one of the most interesting aspects of this study was polling VS surgeons and assessing what they believe was the primary cause for postoperative delayed facial nerve weakness. Approximately 61\% reported this was likely edema tracking along the facial nerve, while only $19 \%$ reported reactivation of latent herpes virus and $18 \%$ suspected vasospasm. Although the mechanism of this remains undetermined, the neural edema appears to be most frequently cited. There are individual case reports of each mechanism being the primary cause. ${ }^{13}$ This number is interestingly very close to the number of respondents utilizing steroid for treatment and may, in fact, impact this delayed facial weakness. Although the majority of respondents have reported use of corticosteroids to prevent postoperative facial weakness, there is a double-blind, placebo-controlled trial demonstrating it did not do so. ${ }^{14}$ In terms of retroviral therapy treating possible reactivation of herpes virus, this was used rarely or never in $75 \%$ of the patients.

Further, the follow-up was extremely variable at which time the first postoperative MRI was most frequently performed. The most common time surgeons reported obtaining the first postoperative MRI scan was either within the first 48 hours (37\%) or between 1 and 6 months postoperatively (also 37\% of the time). In cases of gross total resection, this is perhaps the most sensible place to start with practice standardization to reduce cost and establish standards for follow-up to determine recurrence, especially in cases without evidence of linear or nodular postoperative enhancement. ${ }^{15}$ We did not assess more long-term imaging surveillance patterns among respondents. Future surveys and analysis might benefit from further delving into the differences surgeon's may feel represent acceptable facial nerve outcomes, what is considered useful hearing and what criteria various surgeons use to define near total or subtotal resections (-Table 3).

\section{Conclusion}

Although the perioperative adjuncts to VS surgery are variable, there does appear to be a trend in common practice. Therefore, making surgeons aware of these trends may lead to standardized practice. Alternatively, trials of these variances to instruct us as to which practice patterns truly improve patient outcomes such as perioperative steroid use, DVT prophylaxis, and antibiotic treatment. Awareness is the first step to standardization and practice improvement which this series of articles initiates; there is still a fair amount of work to be done to produce practice standards.

Financial Material and Support

Internal departmental funding was utilized without commercial sponsorship or support.

Conflict of Interest

None.
Institutional Review Board Approval

Institutional Review Board exempted the study.

Acknowledgments

We would like to acknowledge the support of the NASBS research task force, specifically Dr. Devyani Lal, MD.

\section{References}

1 Carlson ML, Glasgow AE, Grossardt BR, Habermann EB, Link MJ. Does where you live influence how your vestibular schwannoma is managed? Examining geographical differences in vestibular schwannoma treatment across the United States. J Neurooncol 2016;129(02):269-279

2 Harner SG, Beatty CW, Ebersold MJ. Headache after acoustic neuroma excision. Am J Otol 1993;14(06):552-555

3 Harner SG, Beatty CW, Ebersold MJ. Impact of cranioplasty on headache after acoustic neuroma removal. Neurosurgery 1995;36 (06):1097-1099, discussion 1099-1100

4 Mosek AC, Dodick DW, Ebersold MJ, Swanson JW. Headache after resection of acoustic neuroma. Headache 1999;39(02):89-94

5 Harner SG, Daube JR, Ebersold MJ, Beatty CW. Improved preservation of facial nerve function with use of electrical monitoring during removal of acoustic neuromas. Mayo Clin Proc 1987;62 (02):92-102

6 Youssef AS, Downes AE. Intraoperative neurophysiological monitoring in vestibular schwannoma surgery: advances and clinical implications. Neurosurg Focus 2009;27(04):E9

7 Aihara N, Murakami S, Takahashi M, Yamada K. Preoperative characteristics of auditory brainstem response in acoustic neuroma with useful hearing: importance as a preliminary investigation for intraoperative monitoring. Neurol Med Chir (Tokyo) 2014;54(04):267-271

8 Battista RA, Wiet RJ, Paauwe L. Evaluation of three intraoperative auditory monitoring techniques in acoustic neuroma surgery. Am J Otol 2000;21(02):244-248

9 Slattery WH III, Francis S, House KC. Perioperative morbidity of acoustic neuroma surgery. Otol Neurotol 2001;22(06):895-902

10 Bratzler DW, Dellinger EP, Olsen KM, et al; American Society of Health-System Pharmacists; Infectious Disease Society of America; Surgical Infection Society; Society for Healthcare Epidemiology of America. Clinical practice guidelines for antimicrobial prophylaxis in surgery. Am J Health Syst Pharm 2013;70(03): 195-283

11 Kartush JM, Cannon SC, Bojrab DI, Graham MD, Schmaltz S, Kemink JL. Use of bacitracin for neurotologic surgery. Laryngoscope 1988;98(10):1050-1054

12 Alshehri N, Cote DJ, Hulou MM, et al. Venous thromboembolism prophylaxis in brain tumor patients undergoing craniotomy: a meta-analysis. J Neurooncol 2016;130(03):561-570

13 Magliulo G, D’Amico R, Celebrini A, Cuiuli G. Postoperative Ramsay-Hunt syndrome after acoustic neuroma resection. Viral reactivation. An Otorrinolaringol Ibero Am 2005;32(03):253-259

14 Bozorg Grayeli A, Ferrary E, Tubach F, et al. Effect of corticosteroids on facial function after cerebellopontine angle tumor removal: a double-blind study versus placebo. Audiol Neurootol 2015;20 (04):213-221

15 Bennett ML, Jackson CG, Kaufmann R, Warren F. Postoperative imaging of vestibular schwannomas. Otolaryngol Head Neck Surg 2008;138(05):667-671 